\title{
THE BALLOON-BORNE LARGE APERTURE SUBMILLIMETER TELESCOPE (BLAST) 2005: CALIBRATION AND TARGETED SOURCES
}

\author{
M. D. P. Truch, ${ }^{1}$ P. A. R. Ade, ${ }^{2}$ J. J. Bock,${ }^{3,4}$ E. L. Chapin, ${ }^{5}$ M. J. Devlin, ${ }^{6}$ S. Dicker,${ }^{6}$ M. Griffin, ${ }^{2}$ J. O. Gundersen, ${ }^{7}$ \\ M. Halpern, ${ }^{5}$ P. C. Hargrave, ${ }^{2}$ D. H. Hughes, ${ }^{8}$ J. Klein, ${ }^{6}$ G. Marsden, ${ }^{5}$ P. G. Martin, ${ }^{9,} 10$ P. Mauskopf ${ }^{2}$ \\ C. B. Netterfield ${ }^{10,11}$ L. Olmi, ${ }^{12,13}$ E. Pascale, ${ }^{11}$ G. Patanchon, ${ }^{14}$ M. Rex, ${ }^{6}$ D. Scott, ${ }^{5}$ C. Semisch, ${ }^{6}$ \\ C. Tucker, ${ }^{2}$ G. S. Tucker, ${ }^{1}$ M. P. Viero, ${ }^{10}$ and D. V. Wiebe ${ }^{11}$ \\ Received 2007 May 29; accepted 2008 February 28
}

\begin{abstract}
The Balloon-borne Large Aperture Submillimeter Telescope (BLAST) operated successfully during a $100 \mathrm{hr}$ flight from northern Sweden in 2005 June (BLAST05). As part of the calibration and pointing procedures, several compact sources were mapped, including solar system, Galactic, and extragalactic targets, specifically Pallas, CRL 2688, LDN 1014, IRAS 20126+4104, IRAS 21078+5211, IRAS 21307+5049, IRAS 22134+5834, IRAS 23011+6126, K3-50, W75N, and Mrk 231. One additional source, Arp 220, was observed and used as our primary calibrator. Details of the overall BLAST05 calibration procedure are discussed here. The BLAST observations of each compact source are described, flux densities and spectral energy distributions are reported, and these are compared with previous measurements at other wavelengths. The 250,350, and $500 \mu \mathrm{m}$ BLAST data can provide useful constraints to the amplitude and slope of the submillimeter continuum, which in turn may be useful for the improved calibration of other submillimeter instruments.
\end{abstract}

Subject headings: balloons — submillimeter — telescopes

Online material: color figures

\section{INTRODUCTION}

The 2005 June flight of the Balloon-borne Large Aperture Submillimeter Telescope (BLAST) incorporated a $2 \mathrm{~m}$ spherical primary mirror and large-format bolometer arrays operating at 250,350 , and $500 \mu \mathrm{m}$. A complete description of the BLAST instrument is given in Pascale et al. (2008). The BLAST wavelengths sample the peak of the spectral energy distribution (SED) for cool dust $(\sim 10-40 \mathrm{~K})$ and are in a regime that is difficult or impossible to access from even the best ground-based sites. As a result, BLAST has the ability to conduct unique Galactic and extragalactic submillimeter surveys with arcminute resolution and high sensitivity. BLAST's primary scientific motivations are to study the angular and redshift distribution and evolution of high-redshift star-forming galaxies and to identify protostellar

\footnotetext{
1 Department of Physics, Brown University, Providence, RI 02912; matthew@ truch.net.

${ }^{2}$ Department of Physics and Astronomy, Cardiff University, Cardiff CF24 3AA, UK

3 Jet Propulsion Laboratory, Pasadena, CA 91109-8099.

4 Observational Cosmology, MS 59-33, California Institute of Technology, Pasadena, CA 91125.

5 Department of Physics and Astronomy, University of British Columbia, Vancouver, BC V6T 1Z1, Canada.

${ }^{6}$ Department of Physics and Astronomy, University of Pennsylvania, Philadelphia, PA 19104.

7 Department of Physics, University of Miami, Coral Gables, FL 33146.

${ }^{8}$ Instituto Nacional de Astrofísica Óptica y Electrónica (INAOE), 72000 Puebla, Mexico.

9 Canadian Institute for Theoretical Astrophysics, University of Toronto, Toronto, ON M5S 3H8, Canada.

10 Department of Astronomy and Astrophysics, University of Toronto, Toronto, ON M5S 3H4, Canada.

11 Department of Physics, University of Toronto, Toronto, ON M5S 1A7, Canada.

12 Istituto di Radioastronomia, I-50125 Florence, Italy.

13 Physics Department, University of Puerto Rico, Rio Piedras Campus, San Juan, PR 00931.

${ }^{14}$ Laboratoire APC, 75205 Paris, France.
}

cores and the earliest stages of star formation within Galactic molecular clouds.

BLAST conducted a $100 \mathrm{hr}$ flight, launching from northern Sweden on 2005 June 12 and landing in northern Canada on 2005 June 16 (BLAST05). One relatively shallow extragalactic field and several large $\left(\sim 8 \mathrm{deg}^{2}\right)$ Galactic fields (see Chapin et al. 2008; Hargrave et al. 2008) were mapped, from which a large number of Galactic prestellar sources were extracted at very high signalto-noise ratio $(\mathrm{S} / \mathrm{N})$. The results obtained from the BLAST flight from Antarctica in 2006 December (BLAST06) will be discussed in future articles.

BLAST05 continuum observations were made with three focal plane arrays consisting of 139,88 , and 43 detectors at 250, 350, and $500 \mu \mathrm{m}$, respectively. The optics and detector layout in the cryostat make simultaneous measurements possible by three arrays, having a common field of view of about $14^{\prime} \times 7^{\prime}$. The detectors are silicon-nitride micromesh ("spiderweb") bolometric detectors coupled with $2 f \lambda$ (maximum optical efficiency; Griffin et al. 2002) spaced feed horn arrays (Turner et al. 2001). The arrays are prototypes of those developed for the SPIRE instrument on Herschel (Griffin et al. 2004).

In this paper we report the observations of compact sources that were targeted by BLAST largely for the purposes of flux and pointing calibration. These sources include Pallas, CRL 2688, K3-50, W75N, Mrk 231, and Arp 220, which have all been well studied in the submillimeter and are commonly used as primary or secondary standards. We also conducted several observations of known bright protostars that were not otherwise included in our wider Galactic plane survey fields, specifically IRAS 20126+4104, IRAS $21078+5211$, IRAS $21307+5049$, IRAS 22134+5834, and IRAS 23011+6126. Finally, we observed the "starless" dense core LDN 1014 that was recently studied with the Spitzer Space Telescope (Young et al. 2004).

Together these observations represent a broad sampling of bright submillimeter sources that were available during the flight, given the strict visibility constraints of the telescope (Pascale et al. 
2008). The planets Mars and Uranus, often used as submillimeter calibrators, were not visible during this flight. Although observations of Saturn were attempted, there were concerns about gain stability because it was relatively close to the direction of the Sun, and it may also have suffered from saturation effects in the detectors, and hence we abandoned attempts to use these particular observations. After carefully assessing the systematic effects in the relevant parts of the SEDs of the other sources, we chose to single out Arp 220 as our primary flux calibrator. Using Arp 220, we determine that the BLAST05 calibration uncertainties are $12 \%, 10 \%$, and $8 \%$ in the 250,350 , and $500 \mu \mathrm{m}$ bands, respectively.

This paper is divided into three main sections. In $\S 2$ we outline the basic reduction steps and characterization of BLAST05 data. In $\S 3$ we discuss the performance of the warm optics in BLAST05 and the impact of the degraded performance on the resolution and sensitivity of the experiment. In $\S 4$ we describe in detail the absolute calibration derived from the primary flux calibrator Arp 220, while in $\S 5$ we summarize the BLAST flux densities and the simple fits of a modified blackbody emission model to the SEDs of each of the targeted sources from the BLAST05 flight.

\section{DATA REDUCTION}

We now describe the main steps in the data reduction and calibration process. Raw data from BLAST consist of a set of bolometer time streams in voltage units, sampled at $100 \mathrm{~Hz}$. The raw bolometer data are first cleaned for postflight analysis. The data are despiked and then deconvolved to remove the electronics filters from the time streams; see Patanchon et al. (2008) for specifics of the cleaning and deconvolution performed on the BLAST data. The cleaned data are combined with a postflight pointing solution (Pascale et al. 2008) to make maps at each frequency, taking advantage of the multiple detectors, as well as significant scan cross linking, to minimize striping due to instrumental drifts.

The bolometers in each array are corrected for relative gains, or flat-fielded, so that meaningful multibolometer maps can be generated. The flat-field corrections are determined using individual maps made for each bolometer from a single pointsource calibrator, in this case CRL 2688 (the brightest pointlike source observed). These scans were designed such that a fully sampled map can be generated from each bolometer individually. For each bolometer, the total flux from the point source is integrated and a flat-fielding coefficient for each bolometer is then calculated as the ratio of the flux from that bolometer and the flux from an arbitrarily chosen reference bolometer. All subsequent maps are generated from time streams that apply the flat-fielding coefficient appropriately to each bolometer.

To calculate the flux density from a point source, we adopted a matched filtering technique similar to that used to extract point sources from several recent extragalactic submillimeter surveys (e.g., Coppin et al. 2006; Scott et al. 2006). A point-spread function (PSF) is generated by stacking and averaging several point sources from various maps in telescope coordinates (azimuth and elevation relative to the telescope). We area-normalize the PSF, $P$, such that

$$
\sum_{x, y} P(x, y) \delta x \delta y=1
$$

where the double sum is over all pixels in the PSF map and $\delta x$ and $\delta y$ are the angular dimensions of a pixel such that the units of $P$ are sr $^{-1}$. In all BLAST maps, square pixels are used so that $\delta x=\delta y$. BLAST maps are calibrated in surface brightness units
$\left(\mathrm{Jy} \mathrm{sr}^{-1}\right)$. A map of a point source, $M(x, y)$, can be modeled by the normalized PSF centered over the source, scaled by its flux density, $S$, or $S P(x, y) \simeq M(x, y)$. If each pixel in $M(x, y)$ has an uncertainty $\sigma(x, y)$, calculated in the mapmaking process (Patanchon et al. 2008), then we can write $\chi^{2}$ for the model, assuming independent noise:

$$
\chi^{2}=\sum_{x, y} \frac{[S P(x, y)-M(x, y)]^{2}}{\sigma^{2}(x, y)} .
$$

Minimizing this $\chi^{2}$ results in the maximum likelihood flux density,

$$
S=\frac{\sum_{x, y}\left[M(x, y) P(x, y) / \sigma^{2}(x, y)\right]}{\sum_{x, y}[P(x, y) / \sigma(x, y)]^{2}} .
$$

This technique uses our knowledge of the beam as a model for the shape of unresolved point sources in BLAST maps. Fitting the amplitude of this template is optimal in an $\mathrm{S} / \mathrm{N}$ sense, and particularly important for measurements of faint sources. This method produces smaller measurement uncertainties than simple aperture photometry, as pixels near the peak signal are weighted more heavily than pixels in the wings of the brightness distribution (aperture photometry weights all pixels equally).

This technique gives a low statistical uncertainty both because we have intrinsically high $\mathrm{S} / \mathrm{N}$ data and also because knowledge of the beam shape is used to weight the uncertainties; however, errors in the PSF will bias the result. To check for a bias, we used simple aperture photometry as a second measure of flux densities for CRL 2688. The difference between the matched filter and aperture photometry flux densities of CRL 2688 is less than $3 \%$. Since the matched filter provides significantly smaller statistical errors, it is used to extract flux densities from all pointlike sources in this paper.

The PSF for each individual bolometer varies slightly across the array. The stacked PSF takes this effect into account for the bulk of the map over which each bolometer contributes approximately equally to the combined signal. However, the extreme edges of the map are only sampled by a fraction of the bolometers and therefore exhibit different effective PSFs. We extract flux densities from maps made with single bolometers using the stacked PSF to assess the error introduced by adopting this incorrect template and find at most a $10 \%$ bias. The bias is maximized at the very edges of the map but affects flux densities measured to within $14^{\prime}$ (width of the array) of the edge of the map along the scan direction (approximately aligned with right ascension) and $7^{\prime}$ (height of the array) in the transverse direction (approximately aligned with declination). None of the measurements discussed in this paper are affected by this bias.

The flat-fielding process was repeated for CRL 2688 observations made during the middle and end of the flight to check for stability. Although no bias or trend is seen, the coefficients for each individual bolometer vary by $5 \% \mathrm{rms}$. This effect averages down when multiple bolometers are used and is negligible in full bolometer array maps as used in this paper. The 5\% scatter in flatfield coefficients appears to be dominated by measurement uncertainties; therefore, this value can be considered a very robust upper limit on the systematic drift in the relative calibration and is insignificant compared to the total calibration error budget given in Table 1.

\subsection{Responsivity Variations}

Variations in bolometer loading (due to changing sky emission, for example) and bolometer base-plate temperature cause changes 
TABLE 1

Calibration Coefficients and Uncertainties for BLAST05

\begin{tabular}{|c|c|c|c|c|c|}
\hline \multirow{2}{*}{$\begin{array}{l}\text { BAND } \\
(\mu \mathrm{m})\end{array}$} & \multirow{2}{*}{$\begin{array}{l}\text { Calibration Coefficient } \\
\qquad\left(\times 10^{12} \mathrm{Jy} \mathrm{V}^{-1}\right)\end{array}$} & \multirow{2}{*}{$\begin{array}{l}\text { UNCERTAINTY } \\
(\%)\end{array}$} & \multicolumn{3}{|c|}{ Pearson Correlation Matrix } \\
\hline & & & $250 \mu \mathrm{m}$ & $350 \mu \mathrm{m}$ & $500 \mu \mathrm{m}$ \\
\hline ................ & 7.61 & 12 & 1 & 0.97 & 0.87 \\
\hline 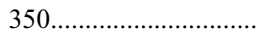 & 3.16 & 10 & $\ldots$ & 1 & 0.96 \\
\hline 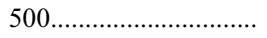 & 1.56 & 8 & $\ldots$ & $\ldots$ & 1 \\
\hline
\end{tabular}

NoTE.-Calibration coefficients, calibration uncertainties, and Pearson correlation matrix, showing the relationship between errors in different bands for BLAST05.

in bolometer responsivities. To characterize and correct for these changes, a calibration lamp ("cal-lamp") is located in the optics box at the center of the reflective Lyot stop (see $\S 3$ of Pascale et al. 2008), allowing for the measurement of these changes. The cal-lamp is pulsed once every $10-15$ minutes during flight. The cal-lamp is of the same design as the one used for SPIRE (Hargrave et al. 2006). It is designed to provide a stable signal over the flight, so any change in cal-lamp amplitude as measured by a bolometer is directly proportional to a change in that bolometer's responsivity. The signal baseline is removed by fitting a line to a $550 \mathrm{~ms}$ segment of data before and after the cal-lamp pulse. A template cal-lamp profile from a raw bolometer time stream is fitted to every cal-lamp pulse in every bolometer. The template is chosen from a typical bolometer, and it has been verified that the template fit amplitude is within $2 \%$ of a square-wave fit. The amplitude of the fit is interpolated over time and inverted to generate a multiplicative scaling cal-lamp time stream for each bolometer. Fluctuations in the responsivity are less than $8 \% \mathrm{rms}$, dominated by diurnal variations due to differences in atmospheric loading and thermal emission from the telescope's changing temperatures. On timescales of a typical source map the fluctuations are less than $2 \%$ rms. A constant responsivity time stream for each bolometer is generated by applying the multiplicative scaling callamp time stream. The large-scale fluctuations are suppressed, and the final responsivity variations are less than $2 \% \mathrm{rms}$.

\section{BLAST05 WARM OPTICS PERFORMANCE}

During the BLAST05 flight, the warm optics (primary and secondary mirrors) did not perform within the specifications. The beams as designed were expected to be close to diffraction limited and approximately Gaussian, $\exp \left(-\theta^{2} / 2 \sigma_{B}^{2}\right)$, with FWHM $=$ $2(2 \ln 2)^{1 / 2} \sigma_{B}=32^{\prime \prime}, 48^{\prime \prime}$, and $64^{\prime \prime}$ at 250,350 , and $500 \mu \mathrm{m}$, respectively. In this case the full width at half-power of the beam, FWHP, is equal to the FWHM, and the beam solid angle can be characterized as $\Omega=\pi \alpha_{D}^{2} /(4 \ln 2)$, with $\alpha_{D}=$ FWHP. The PSFs in all three BLAST bands were measured from multiple observations of the proto-planetary nebula CRL 2688. The in-flight beam shapes are shown in Figure 1. Noting that the central structure in each is on a scale comparable to the diffraction limit, the beams are clearly far from ideal, distributing considerable power into an outer ring (hexagon) of diameter $\sim 200^{\prime \prime}$.

With the preflight predicted noise equivalent flux density (NEFD) of $200 \mathrm{mJy} \mathrm{s}^{1 / 2}$, we would expect $1 \sigma$ surface brightness fluctuations at the nominal resolutions of $\sim \mathrm{NEFD} / \Omega$ or $11,4.7$, and $2.6 \mathrm{MJy} \mathrm{sr}^{-1} \mathrm{~s}^{1 / 2}$ at 250,350 , and $500 \mu \mathrm{m}$, respectively. The measured sensitivities were in fact 8.8, 4.8, and 2.7 $\mathrm{MJy} \mathrm{sr}^{-1} \mathrm{~s}^{1 / 2}$, respectively, which shows that BLAST did not suffer a reduced sensitivity to extended diffuse sources, i.e., detectors worked as planned and optical efficiency was as planned.

There is, however, a marked loss in sensitivity for point sources because of the larger than expected beam solid angle. The FWHP of the degraded beam is $\sim 200^{\prime \prime}$ (somewhat smaller at $500 \mu \mathrm{m}$ ), given the power in the ring. The reduction in point-source sensitivity is $\lesssim(200 / 32)^{2} \simeq 40$ at $250 \mu \mathrm{m}$. This is a pessimistic estimate because there is considerable higher resolution structure in the beam, which one takes advantage of in the PSF fitting described above. Alternatively, one can examine the histogram of pixel brightnesses contributing to the PSF and, working from the brightest, find the beam area that accounts for half the power. We find $\alpha_{D}=186^{\prime \prime}, 189^{\prime \prime}$, and $189^{\prime \prime}$ for 250,350 , and $500 \mu \mathrm{m}$, respectively, and so the sensitivity is reduced by factors of approximately 34,16 , and 9 . Despite this reduction, the targeted sources described in this paper were still detected with high $\mathrm{S} / \mathrm{N}$. Further data from BLAST05 are discussed in Chapin et al. (2008), Hargrave et al. (2008), and other forthcoming papers.

Although it is not critical to the analysis here, we have investigated the origin of the beam degradation. One possibility is misalignment, shifting, and tilting of the secondary and primary

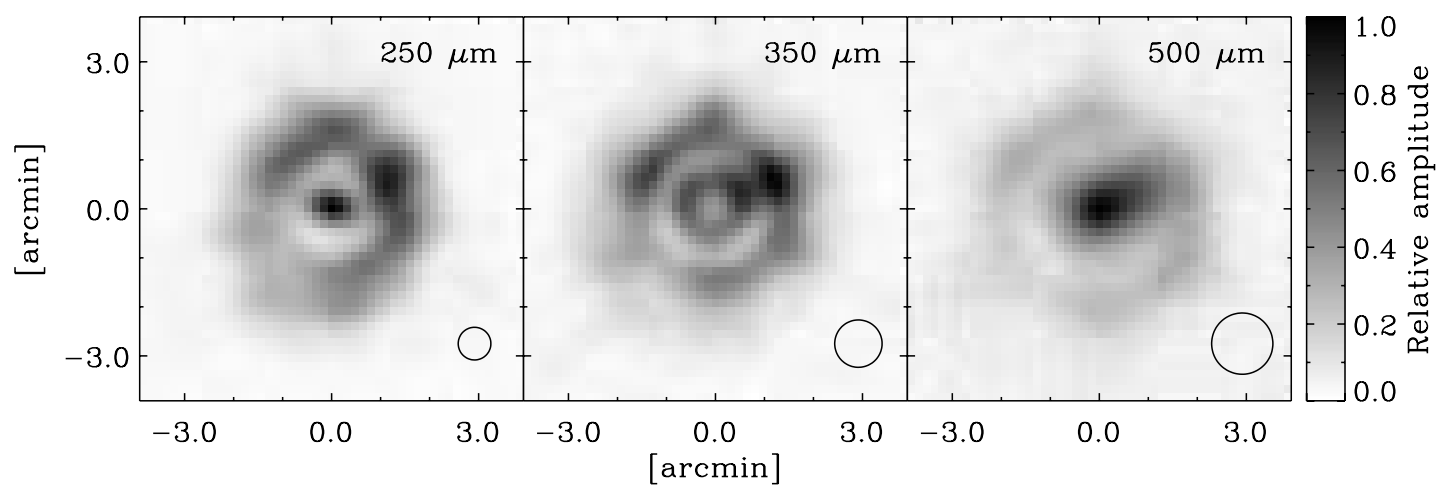

FIG. 1.-PSFs, provided by observations of CRL 2688 ( $\$ 5.2)$, for each of the three wave bands from BLAST05, generated by stacking several point-source maps in

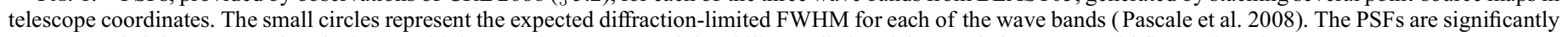
more extended than expected, reducing sensitivity to point sources and the ability to distinguish crowded sources amid fluctuating cirrus. 
with respect to the nominal optical axis. The launch was sufficiently violent to cause the inner frame locking mechanism to fail; the inner frame (which houses the primary, secondary, and camera) violently hit both hardware elevation stops, which could have damaged the telescope, including the secondary support mechanism and the primary itself. From visual inspection of the images (and power spectra) there is clearly information in the beams near the diffraction limit, such as the central dip at $350 \mu \mathrm{m}$, which is not present at the other two wavelengths, and the width of the outer ring. Since these structures are suggestive of a problem in the telescope, we performed numerous simulations with the optical design software ZEMAX ${ }^{15}$ In these simulations, we shifted and/or tilted all of the optical elements from their nominal position but were unable to reproduce the distinctive features in the beams at all three wavelengths simultaneously. There appears to have been an optical problem beyond out-of-focus or out-ofalignment optics. The hexagonal shape of the beam does suggest damage to the carbon fiber primary itself, which was constructed from six panels (segments). In addition to effects of the violent launch, another contributing factor could have been the light rain the payload endured before launch. Carbon fiber becomes particularly weak in a high-humidity environment, possibly leading to delamination of the six panels either before or during the launch. Furthermore, there was the usual freeze-thaw cycle on ascent, where the ambient temperature dropped as low as $-65^{\circ} \mathrm{C}$. Further analysis of the PSF (including Fourier transform) was not performed as phase information is required for proper analysis; as the mirror was fully destroyed on landing, there is little use in debugging the system further.

\section{ASTRONOMICAL FLUX CALIBRATION}

The primary scientific goals of the BLAST experiment demanded a flux calibration accuracy of better than $10 \%$ in all three BLAST passbands. Achieving this was complicated by the variable sky visibility of BLAST due to the unstable projected latitude of the telescope gondola during the flight, as well as the restrictions on visibility due to the Sun and Moon avoidance criteria, the orientation of Sun shields and other baffling, and the elevation range $\left(25^{\circ}-60^{\circ}\right)$ of the gondola's inner frame (Pascale et al. 2008). Consequently, BLAST had only limited access to the calibration sources commonly used at submillimeter and farinfrared (FIR) wavelengths.

Since the ecliptic plane was not visible during the BLAST05 flight, no absolute flux calibration of BLAST could be determined from observations of Uranus or Mars, for which model SEDs are known to have systematic uncertainties $<5 \%$ at submillimeter wavelengths (Griffin \& Orton 1993; Wright 2007). The preflight strategy for achieving a $10 \%$ calibration accuracy, recognizing the above visibility constraints, therefore forced us to identify other Galactic and extragalactic sources that could act as primary and secondary calibrators, with the following requirements: (1) availability throughout the flight; (2) already considered, in some cases, as secondary calibrators for ground-based submillimeter telescopes and FIR satellites; (3) well-constrained SEDs in the FIR to millimeter wavelength regime, enabling accurate interpolation of the band-averaged flux densities at BLAST wavelengths; and (4) bright ( $\gg 1$ Jy at $500 \mu \mathrm{m}$ ) and compact sources (with respect to the BLAST beam size, $<20^{\prime \prime}$ ) that reside in regions with minimal spatial structure in the Galactic foregrounds or backgrounds, allowing accurate subtraction of any extended emission. Given these criteria, we scheduled regular observations

15 See http://www.zemax.com.

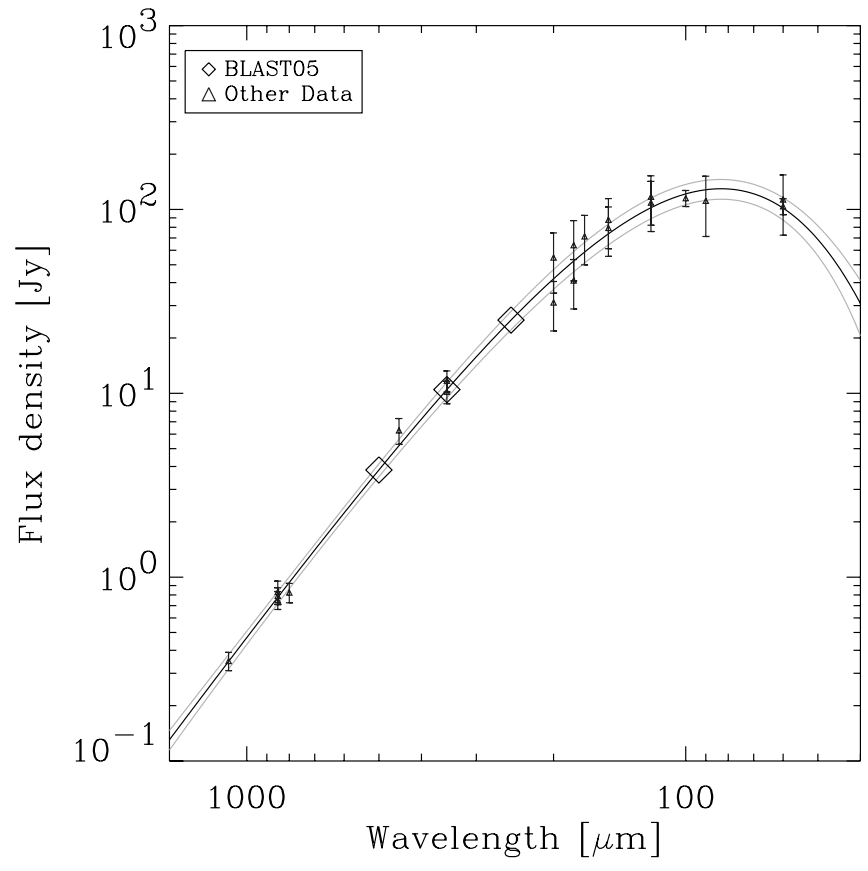

FIG. 2.-SED of Arp 220, the absolute flux calibrator for BLAST05. The best-fit model (thick solid line) was constrained by the published data (triangles; discussed in $\S 4.1$ ) and excluded BLAST05 measurements. The gray lines show the $68 \%$ confidence interval, estimated from 100 Monte Carlo simulations, about the best-fit model. Black diamonds indicate the model predictions for BLAST05 at 250,350 , and $500 \mu \mathrm{m}$. The uncertainties associated with these predictions are $11 \%, 10 \%$, and $8 \%$ in each band, respectively. The best-fit parameters of the single-temperature dust model are given in Table 3. [See the electronic edition of the Journal for a color version of this figure.]

throughout the flight of bright embedded protostellar sources and compact $\mathrm{H}$ II regions within the W58 and Cygnus X molecular cloud complexes (including K3-50, W75N, and DR 21), a postasymptotic giant branch (post-AGB) star with planetary nebula (CRL 2688), the asteroid 2 Pallas, and two ultraluminous infrared galaxies (ULIRGs), Arp 220 and Mrk 231.

In the following subsection we justify our selection of the ULIRG Arp 220 as our primary calibration source for the BLAST05 flight.

\subsection{Arp 220-a Primary Flux Calibrator for BLAST05}

Arp 220 (Arp 1966) is a well-studied ULIRG at redshift $z=$ 0.018 with an FIR luminosity of approximately $2 \times 10^{12} L_{\odot}$. The submillimeter emission is believed to be due to dust heated primarily by active star formation (at a rate of $\sim 200 M_{\odot} \mathrm{yr}^{-1}$ ) and to a lesser extent by an embedded active galactic nucleus (AGN). This emission is confined to a compact ( $<2^{\prime \prime}$ diameter) region, as traced through interferometric imaging, by the distribution of the $1.3 \mathrm{~mm}$ dust continuum, and the molecular gas in the $\mathrm{CO}(2-1)$ line (Scoville et al. 1997) that fuels the starburst activity. Arp 220 resides far from the Galactic plane $\left(b=+53^{\circ}\right)$, and extended foreground Galactic contamination as seen in the $100 \mu \mathrm{m}$ map (Schlegel et al. 1998) does not affect the measured SED. Arp 220 is therefore a pointlike source as observed by BLAST, as well as most ground-based and space-borne FIR to millimeter telescopes, making the construction of the FIRmillimeter SED insensitive to the aperture sizes of the photometric measurements (Fig. 2).

The quoted measurement errors for the ground-based 350$1100 \mu \mathrm{m}$ photometry of Arp 220 are $\leq 10 \%$, while the absolute calibration uncertainties are typically $10 \%-20 \%$. In the case of 
shorter wavelength FIR measurements from ISOPHOT at 60 $200 \mu \mathrm{m}$ (Klaas et al. 2001) and 120-200 $\mu \mathrm{m}$ (Spinoglio et al. 2002), the absolute calibration of ISOPHOT, which is estimated to be $\sim 30 \%$, also dominates the total photometric accuracy. More precise photometry, however, at 60 and $100 \mu \mathrm{m}$ is available from the IRAS Revised Bright Galaxy Sample (RBGS; Sanders et al. 2003), with statistical uncertainties that have been quoted at the $\sim 0.1 \%$ level. The difference between the absolute calibration of the RBGS and the original IRAS Bright Galaxy Samples ranges from $5 \%$ to $25 \%$, and our analysis therefore assumes an additional $10 \%$ absolute calibration uncertainty on the IRAS flux densities. As we show below, combining all the above data and accounting for systematic uncertainties, we have been able to constrain a simple SED model of thermal dust emission that allows interpolated band-averaged flux densities of Arp 220 to be estimated across the BLAST bandpasses with an acceptable $\sim 10 \%$ uncertainty (Fig. 2).

In order to predict the BLAST band-averaged fluxes of Arp 220, a single-temperature modified blackbody SED of the form $S_{\nu}=A\left(\nu / \nu_{0}\right)^{\beta} B_{\nu}(T)$ was first fitted to existing submillimeter and FIR observations described previously. In the above expression, $S_{\nu}$ is the flux density, $A$ is the amplitude, $\beta$ is the emissivity index of the radiating dust grains, $\nu_{0}$ is fixed at $c / 250 \mu \mathrm{m}$, and $B_{\nu}(T)$ is the Planck function for a blackbody with temperature $T$.

The smooth SED is fitted to the data using $\chi^{2}$ minimization. In addition to the measurement errors quoted in $\S 4.1$, we have assumed an additional correlated error of $5 \%$ for all of the photometry, since the bulk of these instruments were calibrated using the same Uranus SED, which is known to have an uncertainty of $\sim 5 \%$ (Griffin \& Orton 1993). Furthermore, we have been careful to account for correlated errors using the full data covariance matrix,

$$
\chi^{2}=(\tilde{\boldsymbol{S}}-\boldsymbol{S}) C^{-1}(\tilde{\boldsymbol{S}}-\boldsymbol{S})^{T},
$$

where $\boldsymbol{S}$ is the vector of measured flux densities and $\tilde{\boldsymbol{S}}$ are the model predictions. The diagonal elements of $C$ give the variances, $\sigma_{i}^{2}$, calculated as the quadrature sums of the photometric and calibration uncertainties described above. The off-diagonal elements of $C$ are calculated as follows. For each pair of measurements taken with the same group and same instrument, $i$ and $j$, calibration uncertainties are assumed to be completely correlated such that $C_{i j}=\sigma_{i}^{c} \sigma_{j}^{c}$, where $\sigma^{c}$ is the standard deviation of the calibration error component for the measurement. Monte Carlo simulations are performed to characterize the range of models that are consistent with the data. One thousand mock data sets are generated by adding realizations of correlated Gaussian noise described by $C$ to the measured flux densities $S$. Each one of these data sets is then refitted using equation (4). The best-fit model ( $T=41.7 \mathrm{~K}$ and $\beta=1.3$ ) for the observed SED and its uncertainties are shown in Figure 2.

The band-averaged flux densities for each BLAST wavelength are obtained for each SED model fit as

$$
\tilde{S}_{B}=\int T(\nu) \tilde{S}(\nu) d \nu,
$$

where we use tildes to represent model quantities. Here the normalized filter transmission profiles $T(\nu)$ are measured empirically using a Fourier transform spectrometer (Pascale et al. 2008). From these 1000 sets of band-averaged fluxes, we obtain estimates of variances of the calibrator brightnesses in each band, $\left\langle\sigma_{i}^{2}\right\rangle$, as well as the correlated error between the fluxes in each band, $\left\langle\sigma_{i} \sigma_{j}\right\rangle$.
Maximum likelihood estimates for the flux densities of all BLAST05 sources are obtained from equation (3) using uncalibrated maps of Arp 220 in units of V. Multiple observations of CRL 2688 were made throughout the flight, allowing us to estimate systematic gain variations not traced by the internal cal-lamp pulses at the $3 \%$ level. Since this gain variation is significantly lower than the calibrator flux uncertainty estimated above, we calculate single calibration coefficients $c_{i}$ in each band for the entire flight as the ratio of the band-averaged fluxes to the raw fluxes from our maps. Note that this calibration depends explicitly on the assumption of a smooth thermal SED that neglects the effects of molecular emission lines.

This problem has been examined in detail for SCUBA $850 \mu \mathrm{m}$ continuum observations of a variety of Galactic objects. Friesen et al. (2005) conclude that the line contamination is typically $<10 \%$ in hot Galactic molecular cores, and a similar conclusion was reached by Johnstone et al. (2003) in a study of protostellar sources in Orion, although in some exceptional cases the line contamination of the $850 \mu \mathrm{m}$ continuum fluxes in the most energetic sources reached levels $>50 \%$. Similarly, line contamination, due exclusively to $\mathrm{CO}(3-2)$ emission, has been measured to be $<10 \%$ in $850 \mu \mathrm{m}$ continuum fluxes, for $\sim 60 \%$ of the 45 local galaxies observed by Yao et al. (2003). Unfortunately, there is almost no information on the strengths of possible line contamination in the BLAST passbands, including redshifted molecular lines at $\ll 300 \mu \mathrm{m}$, and thus we assume that $<10 \%$ line contamination is present in the BLAST data.

Including all of these sources of uncertainty, the final percentage errors $(8 \%-12 \%)$ in the absolute flux calibration and a Pearson correlation matrix showing the relationship between errors in the different bands are given in Table 1 . The calibration coefficients are used to convert raw (voltage) maps into calibrated (Jy) maps. All astronomical analysis is performed on the calibrated maps.

\section{BLAST05 OBSERVATIONS OF BRIGHT SOURCES}

In this section we summarize the calibrated BLAST observations of all the bright targets presented in this paper, paying attention to the level of agreement between their calibrated BLAST fluxes and the predictions from an interpolation of their measured SEDs. Although these bright sources were selected to provide checks on the consistency of the calibration throughout the flight and were not considered to be part of the scientific program, we derive some of the physical properties (temperatures, FIR luminosities, dust masses) that can be obtained from a simple model of thermal dust emission that has been fitted to the improved spectral coverage of their SEDs $(\S 5.1 .1)$

In addition to conducting large-area surveys of Galactic fields undergoing active star formation (Chapin et al. 2008), BLAST05 observed a total of nine bright, individual pointlike sources (Pallas, CRL 2688, IRAS 20126+4104, IRAS 21078+5211, IRAS 21307+ 5049, IRAS 22134+5834, IRAS 23011+6126, Mrk 231, and Arp 220), complemented by observations of more extended starforming structures toward W75N in Cyg X and K3-50 in W58, as part of the overall calibration strategy. Although not used explicitly as a calibration source, we also present in this paper the BLAST05 observations of the compact low-mass protostar L1014-IRS toward the nearby Galactic dark cloud LDN 1014.

All BLAST05 calibration targets are in the northern hemisphere $\left(23^{\circ}<\right.$ decl. $<61^{\circ}$; Table 2$)$. BLAST therefore provides valuable new photometric data at intermediate wavelengths to complement those obtained in the FIR and the (sub)millimeter regime. 
TABLE 2

Flux Densities of BLASt05 Targeted Sources

\begin{tabular}{|c|c|c|c|c|c|}
\hline \multirow[b]{2}{*}{ NAME } & \multirow{2}{*}{$\begin{array}{c}\text { R.A. }^{\mathrm{a}} \\
(\mathrm{J} 2000.0)\end{array}$} & \multirow{2}{*}{$\begin{array}{c}\text { DECL. }^{\mathrm{a}} \\
(\mathrm{J} 2000.0)\end{array}$} & \multicolumn{3}{|c|}{ Flux Density (Jy) } \\
\hline & & & $250 \mu \mathrm{m}$ & $350 \mu \mathrm{m}$ & $500 \mu \mathrm{m}$ \\
\hline Pallas ${ }^{\mathrm{b}} .$. & & & $11.6 \pm 1.5$ & $6.3 \pm 0.7$ & $3.7 \pm 0.3$ \\
\hline CRL 2688 & 210218.75 & +364137.8 & $113 \pm 14$ & $49 \pm 5$ & $21.6 \pm 1.7$ \\
\hline LDN 1014 & 212406 & +49 59.'1 & $22.4 \pm 2.7$ & $16.9 \pm 1.7$ & $8.9 \pm 0.7$ \\
\hline IRAS $20126+4104 \ldots \ldots \ldots \ldots \ldots \ldots$ & 201425.1 & +411332 & $590 \pm 71$ & $256 \pm 26$ & $97 \pm 8$ \\
\hline IRAS $21078+5211 \ldots \ldots$ & 210925.2 & +522344 & $1300 \pm 160$ & $590 \pm 60$ & $260 \pm 21$ \\
\hline IRAS $21307+5049$ & 213231.5 & +510222 & $102 \pm 12$ & $46 \pm 5$ & $18 \pm 1.5$ \\
\hline IRAS $22134+5834 \ldots \ldots \ldots \ldots \ldots \ldots$ & 221509.1 & +584909 & $418 \pm 50$ & $208 \pm 21$ & $82 \pm 7$ \\
\hline IRAS $23011+6126 \ldots \ldots \ldots \ldots$ & 230313.9 & +614221 & $95 \pm 12$ & $59 \pm 6$ & $31 \pm 2.5$ \\
\hline $\mathrm{K} 3-50 \mathrm{~A}^{\mathrm{c}}$ & 200145.6 & +333242 & $2100 \pm 270$ & $590 \pm 67$ & $270 \pm 26$ \\
\hline K3-50C & 200154.2 & +333415 & $1870 \pm 240$ & $1000 \pm 110$ & $370 \pm 35$ \\
\hline $\mathrm{W} 75 \mathrm{~N}^{\mathrm{d}} \ldots$ & 203836.5 & +423735 & $4500 \pm 540$ & $2000 \pm 200$ & $730 \pm 60$ \\
\hline DR $21(\mathrm{OH})^{\mathrm{d}}$.. & 203900.9 & +422238 & $9100 \pm 1400$ & $4600 \pm 660$ & $2540 \pm 330$ \\
\hline DR $21^{\mathrm{d}}$ & 203901.1 & +421943 & $8800 \pm 1400$ & $3900 \pm 550$ & $920 \pm 120$ \\
\hline Mrk 231 & 125614.23 & +565225.2 & $6.0 \pm 0.8$ & $1.5 \pm 0.3$ & $\mathrm{e}$ \\
\hline Arp $220^{\mathrm{f}}$ & 153457.21 & +233009.5 & 24.2 & 9.8 & 3.9 . \\
\hline
\end{tabular}

Notes.-Flux densities and associated uncertainties for BLAST05 targeted sources in the 250,350 , and $500 \mu \mathrm{m}$ bands. Units of right ascension are hours, minutes, and seconds, and units of declination are degrees, arcminutes, and arcseconds. Quoted flux densities have been color-corrected. The uncertainties include the estimated contributions from calibration uncertainty, as well as instrumental noise.

a Positions are nominal as given by SIMBAD.

b Pallas flux densities are average values from four observations taken at the following Julian Dates: 2,453,534.26; 2,453,534.29; $2,453,535.22 ;$ and $2,453,535.40$.

c The K3-50 region is resolved into two sources by BLAST05.

d The $\mathrm{W} 75 \mathrm{~N}$ region is resolved into three sources by BLAST05.

${ }^{\mathrm{e}}$ Mrk 231 was not detected at $500 \mu \mathrm{m}$ by BLAST05.

${ }^{\mathrm{f}}$ Arp 220 is the absolute flux calibrator for BLAST05. Flux densities presented here should thus be considered as predictions based on a model fit to data from other instruments. Uncertainties in this model are given in $\S 4.1$.

With the advantage of simultaneous observations at 250, 350, and $500 \mu \mathrm{m}$, BLAST measures the thermal emission of these Galactic and extragalactic objects with a high flux calibration accuracy in a wavelength regime that traces the spectral curvature at wavelengths longer than the spectral peak and shorter than the Rayleigh-Jeans limit for cold dust $(<50 \mathrm{~K})$. In terms of modeling the SEDs, this short submillimeter wavelength regime sampled by BLAST is critical for unlocking the degeneracies between the effect of dust temperature, opacity, and emissivity index on the overall FIR-millimeter emission. The SPIRE instrument, using similar bolometer arrays and identical filters to BLAST, following the future launch of Herschel (Griffin et al. 2004), will extend this work with greater sensitivity and spatial resolution.

It is not the purpose of this paper to provide a detailed account of physical properties and the nature of each bright source observed in BLAST05. Thus, we only discuss briefly in the following subsections the collective results obtained from these BLAST observations at 250,350 , and $500 \mu \mathrm{m}$.

\subsection{Spectral Energy Distributions}

Flux densities at 250, 350, and $500 \mu \mathrm{m}$ for all the above point sources are extracted using the matched filter technique (eq. [3] outlined in $\S 2$ ), while for extended or blended sources at the resolution of BLAST05 (e.g., toward K3-50 and W75N), we convolve the PSF with a simple model, which is then fitted to the data. In the latter case the model consists of multiple Gaussian sources, where we allow the amplitude, width, and position of the Gaussians to vary as parameters of the fit.

Since the BLAST filters have large spectral widths, and colors sampled by the filters are a strong function of temperature (especially for $T \lesssim 25 \mathrm{~K}$ ), a correction must be made to calculate monochromatic flux densities. Either a color correction may be applied to the band-averaged flux densities, or effective filter wavelengths for each object may be quoted. We choose the former and quote effective flux densities at precisely 250,350 , and $500 \mu \mathrm{m}$. Once the SED has been fitted to the BLAST data by minimizing $\chi^{2}$, to obtain the best-fit dust temperature and emissivity index, this is used to calculate the correction

$$
S(\nu)=S_{B} \frac{\tilde{S}(\nu)}{\tilde{S}_{B}},
$$

where $S_{B}$ is the band-averaged BLAST flux measurement, $\tilde{S}(\nu)$ is the SED model flux density (evaluated at 250,350 , and $500 \mu \mathrm{m}$ ), and $\tilde{S}_{B}$ is the SED model band-averaged flux. The BLAST colorcorrected flux densities for all bright sources presented in this paper are given in Table 2.

\subsubsection{Single-Temperature Cold Dust Models}

Incorporating the BLAST measurements into the existing FIRmillimeter wavelength $(\sim 50-2000 \mu \mathrm{m})$ SEDs for each source, we fitted only a single-temperature dust component while recognizing that any model that utilizes shorter wavelength mid-IR data must naturally include thermal emission from hot dust $(>100 \mathrm{~K})$. This decision is justified in the context of deriving the bandaveraged BLAST fluxes, which require only a smooth and accurate fit to the SED over the restricted wavelength range of the BLAST filters (Pascale et al. 2008). Although alternative and more complex models (e.g., two-temperature components) can be fitted to the same FIR-millimeter data, the difference in the quality (e.g., minimized $\chi^{2}$ ) of the fit is not significant when compared to the single-temperature model.

As previous studies of the FIR-millimeter SEDs of local galaxies and ULIRGs have shown (e.g., Eales et al. 2000; Lisenfeld et al. 2000), relying on limited photometric data, with typically three to five measurements in the FIR (from IRAS and ISO) and 
TABLE 3

Single-Temperature SED Best-Fit Parameters of Blast05 Targeted Sources

\begin{tabular}{|c|c|c|c|c|c|c|}
\hline Name & $\begin{array}{c}T \\
(\mathrm{~K})\end{array}$ & $\beta$ & $\begin{array}{c}S_{\mathrm{FIR}} \\
\left(\mathrm{W} \mathrm{m}^{-2}\right)\end{array}$ & $\begin{array}{l}\text { Distance } \\
(\mathrm{kpc})\end{array}$ & $\begin{array}{l}L_{\mathrm{FIR}} \\
\left(L_{\odot}\right)\end{array}$ & $\begin{array}{l}M_{\text {dust }} \\
\left(M_{\odot}\right)\end{array}$ \\
\hline Pallas $^{\mathrm{a}}$. & 50.2 & $0.2 \pm 0.2$ & $1.2 \times 10^{-12}$ & & & \\
\hline 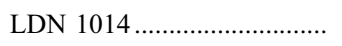 & $12 \pm 3$ & $1.8 \pm 0.5$ & $(3.3 \pm 1.2) \times 10^{-13}$ & 0.2 & $0.38 \pm 0.14$ & $(3.7 \pm 3.5) \times 10^{-3}$ \\
\hline IRAS $20126+4104^{\mathrm{a}} \ldots \ldots \ldots \ldots \ldots$ & 41 & $1.1 \pm 0.2$ & $9.3 \times 10^{-11}$ & 1.7 & $4.0 \times 10^{4}$ & 0.67 \\
\hline IRAS $21078+5211 \ldots \ldots \ldots \ldots . .$. & $33.7 \pm 2.0$ & $1.0 \pm 0.2$ & $(1.2 \pm 0.1) \times 10^{-10}$ & 1.65 & $(1.0 \pm 0.1) \times 10^{4}$ & $0.29 \pm 0.05$ \\
\hline IRAS $23011+6126 \ldots \ldots \ldots \ldots \ldots$ & $27.8 \pm 2.8$ & $0.6 \pm 0.2$ & $(4.3 \pm 0.5) \times 10^{-12}$ & 0.73 & $71 \pm 9$ & $0.01 \pm 0.007$ \\
\hline 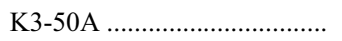 & $39.7 \pm 2.8$ & $1.7 \pm 0.2$ & $(6.2 \pm 0.5) \times 10^{-10}$ & 8.5 & $(1.4 \pm 0.1) \times 10^{6}$ & $8.1 \pm 1.4$ \\
\hline 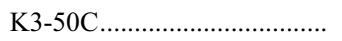 & $9.3 \pm 1.6$ & $3.9 \pm 0.5$ & $(2.3 \pm 0.8) \times 10^{-11}$ & 8.5 & $(5.2 \pm 2.0) \times 10^{4}$ & $(1.2 \pm 1.1) \times 10^{3}$ \\
\hline $\mathrm{W} 75 \mathrm{~N}$ & $65^{\mathrm{b}}$ & $0.8 \pm 0.2$ & $(2.5 \pm 1.3) \times 10^{-9}$ & 3 & $(7.1 \pm 3.6) \times 10^{5}$ & $1.0 \pm 0.1$ \\
\hline DR $21(\mathrm{OH}) \ldots \ldots \ldots \ldots \ldots \ldots \ldots . .$. & $40^{\mathrm{c}}$ & $0.5 \pm 0.3$ & $(7.3 \pm 3.3) \times 10^{-10}$ & 3 & $(2.1 \pm 0.9) \times 10^{5}$ & $4.9 \pm 0.7$ \\
\hline 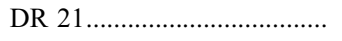 & $29.2 \pm 3.0$ & $2.3 \pm 0.2$ & $(1.7 \pm 0.3) \times 10^{-9}$ & 3 & $(4.9 \pm 0.8) \times 10^{5}$ & $8.7 \pm 3.0$ \\
\hline 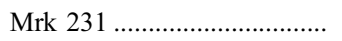 & $43.9 \pm 2.5$ & $1.5 \pm 0.1$ & $(1.8 \pm 0.2) \times 10^{-12}$ & 175000 & $(1.6 \pm 0.1) \times 10^{12}$ & $(6.4 \pm 0.8) \times 10^{6}$ \\
\hline
\end{tabular}

Notes.-Parameters for a single-temperature modified blackbody fit to the SED. Tand $\beta$ are the best fit to the BLAST05 and other data as indicated in the text. $S_{\text {FIR }}$ is the total flux from the modified blackbody fit. Errors are from 100 Monte Carlo simulations of the fit. Distances given are from references indicated in $\S 5$. FIR luminosity is based on these adopted distances. $M_{\text {dust }}$ is based on the formula $M_{\text {dust }}=\left(S_{\nu} D^{2}\right) /\left[\kappa B_{\nu}(T)\right]$, with an assumed value of 10 for $\kappa$ (see Chapin et al. 2008).

${ }^{a}$ The data included in the fit do not accurately constrain the temperature, so no error bars are given.

${ }^{b}$ Data from Harvey et al. (1977) suggest a temperature of $65 \mathrm{~K}$, which is used as a constraint in the fit.

c Data from Harvey et al. (1977) suggest a temperature of $40 \mathrm{~K}$, which is used as a constraint in the fit.

${ }^{d}$ Arp 200 is the primary calibrator for BLAST05. This is our best fit to the other data, which we use to calibrate the BLAST photometry.

(sub)millimeter data (at $850 \mu \mathrm{m}$ and $1.2 \mathrm{~mm}$ data), these SEDs can be well represented by a single dust temperature in the range of $25-50 \mathrm{~K}$. Similar conclusions have been reached from the studies of dust in the Galactic interstellar medium. Even with the addition of ground-based 350 and $450 \mu \mathrm{m}$ data (e.g., Benford 1999; Hunter et al. 2000; Dunne \& Eales 2001; Beelen et al. 2006; Coppin et al. 2008), single-temperature fits work fairly well, and there is typically no need to fit multiple-temperature components to SEDs unless one also brings in data at $\lambda \ll 100 \mu \mathrm{m}$. More complicated fits would only be demanded with higher fidelity spectral measurements covering a much wider range of wavelengths.

Given this, we show the mid-IR to millimeter wavelength SEDs of all the bright compact sources observed by BLAST05 and their best-fit single-temperature models in Figures 2-9, and we present the best-fit model parameters in Table 3 .

Adopting a distance to each source ( $\S 5.8$ ), we determine the FIR luminosity and dust mass from the SED fits, recognizing that the adoption of two or more temperature components would modify the dust mass estimate. Determination of bolometric fluxes and dust masses follows the procedures described in Chapin et al. (2008). Uncertainties in quantities derived from the SED fits in Table 3 are obtained from Monte Carlo simulations similar to those described in $\S 4.1$, which now include BLAST data and their correlated calibration uncertainties. For each mock data set the quantity in question is derived from the model fit. These values are then placed in histograms in order to extract means and $68 \%$ confidence intervals. Further details on these Monte Carlo simulations are provided by Chapin et al. (2008).

\subsection{CRL 2688}

CRL 2688 is a post-AGB star with a proto-planetary nebula (the "Egg"; Price \& Murdock 1983), and it is considered an archetypal object with which to study the evolution of AGB red giants into bipolar planetary nebulae. The distance to CRL 2688 is not well known but is estimated to be approximately $1.25 \mathrm{kpc}$ (Crampton et al. 1975; Cohen \& Kuhi 1977). Despite residing in the Galactic plane, CRL 2688 exhibits compact submillimeter emission and sufficient contrast relative to its surroundings to provide a commonly used secondary calibrator (e.g., Sandell 1994; Jenness et al. 2002). The submillimeter emission arises from a dust shell with an extent of approximately $5^{\prime \prime}$ (Jenness et al. 2002), making it pointlike for BLAST. CRL 2688 was visited regularly throughout the flight; thus, the observations were useful for tracking the PSF shape (Fig. 2) and relative antenna gain variations, making it our primary flat-fielding calibrator.

Fainter surface brightness shells around CRL 2688 have also been observed at $120-180 \mu \mathrm{m}$ with $I S O$ at a radial distance of $\sim 150^{\prime \prime}$ and $300^{\prime \prime}$ (Speck et al. 2000), although this has recently been disputed with Spitzer MIPS observations (Do et al. 2005). In any case, these shells would be large compared to the BLAST beam and of uniform surface brightness, such that any faint emission would be removed from the measured fluxes through our baseline subtraction. No long-term submillimeter variability has been detected in submillimeter observations with SCUBA (Jenness et al. 2002), and hence archival data can be combined to perform our calibrations.

The mid-IR to FIR SED has been accurately measured by ISO LWS between 43 and $194 \mu \mathrm{m}$ (Cox et al. 1996). At submillimeter wavelengths, Jenness et al. (2002) report the integrated fluxes derived from SCUBA maps at 450 and $850 \mu \mathrm{m}$, over a $40^{\prime \prime}$ aperture, which exceed the earlier single-beam peak fluxes of Sandell (1994). Combining these data with the new BLAST measurements (Fig. 3), we find an acceptable fit to a singletemperature modified blackbody model, deriving a dust temperature of $210 \mathrm{~K}$ and emissivity index of $0.4 \pm 0.2$. The SED of CRL 2688 in the FIR-submillimeter regime traced by BLAST is not as accurately constrained as that of Arp 220 as there are no FIR measurements with either IRAS or Spitzer MIPS due to saturation. We derive uncertainties in the BLAST fluxes of CRL 2688 that range from $\sim 10 \%$ at $500 \mu \mathrm{m}$ to $\sim 20 \%$ at $250 \mu \mathrm{m}$.

\subsection{Pallas}

2 Pallas is the third largest asteroid, with a diameter of $530 \mathrm{~km}$ (Mitchell et al. 1996). It has a well-determined, eccentric orbit 


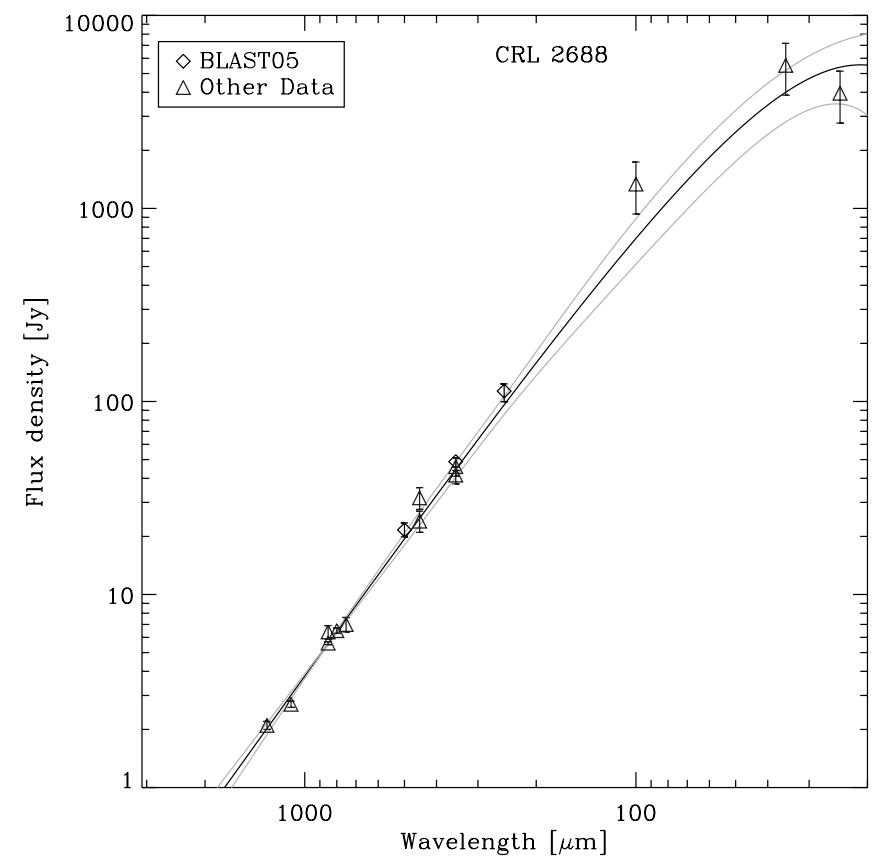

FIG. 3.-CRL 2688 SED, showing BLAST data and best-fitting models. Symbols and lines are as those described in Fig. 2. ISO LWS data between 43 and $194 \mu \mathrm{m}$ are presented by Cox et al. (1996). Other data are taken from Sandell (1994), Jenness et al. (2002), and Omont et al. (1995). [See the electronic edition of the Journal for a color version of this figure.]

with a semimajor axis between 2.1 and 3.4 AU. At the time of the BLAST observations, 2 Pallas was at a distance of $2.2 \mathrm{AU}$. With an angular size $<1^{\prime \prime}$, Pallas is pointlike to BLAST. Errors in the derived flux densities of Pallas at BLAST wavelengths are $5 \%-10 \%$ and are due mostly to uncertainties in the size, shape, and albedo of the asteroid. The current best estimates for the physical properties of Pallas come from other submillimeter and IR flux density measurements integrated into the "thermophysical" model (TPM) of Müller \& Lagerros (2002). The TPM has an uncertainty of around $5 \%-10 \%$ for integrated fluxes in the ISO LWS band. The absolute calibration of the model has a supposed accuracy of $\sim 10 \%$ in the longer wavelength BLAST bands, comparing predictions with measurements from the JCMT $(850 \mu \mathrm{m})$ and CSO $(350 \mu \mathrm{m})$ (T. Müller 2005, private communication). Although this uncertainty is similar to that for the SED of Arp 220, Arp 220 was adopted as the absolute flux calibrator over Pallas since it is brighter, and its SED does not vary over the duration of the flight. Changes in apparent brightness due to orbital effects are negligible on the timescale of the individual BLAST observations (T. Müller 2005, private communication), and the variations due to the $7.8 \mathrm{hr}$ rotational period of 2 Pallas are less than $7 \%$ during the entire BLAST flight (T. Müller 2005, private communication). Although the TPM contains a full shape description and spin model (enabling SED predictions at any epoch), the uncertainty in the absolute calibration of the model flux is comparable to the predicted maximal brightness variations during the BLAST flight. For this reason, in Table 2 we have simply averaged together our four sets of Pallas observations, and we plot only the BLAST photometry points in Figure 4, with no attempt to relate these to other data at significantly different epochs. The TPM predicts Pallas fluxes of 13.2, 6.8, and 3.4 Jy, which are consistent with the BLAST05 measurments, differing by $14 \%$, $8.6 \%$, and $7.3 \%$ at 250,350 , and $500 \mu \mathrm{m}$, respectively. The submillimeter spectral index $\beta$ in the SED of Pallas, as measured by

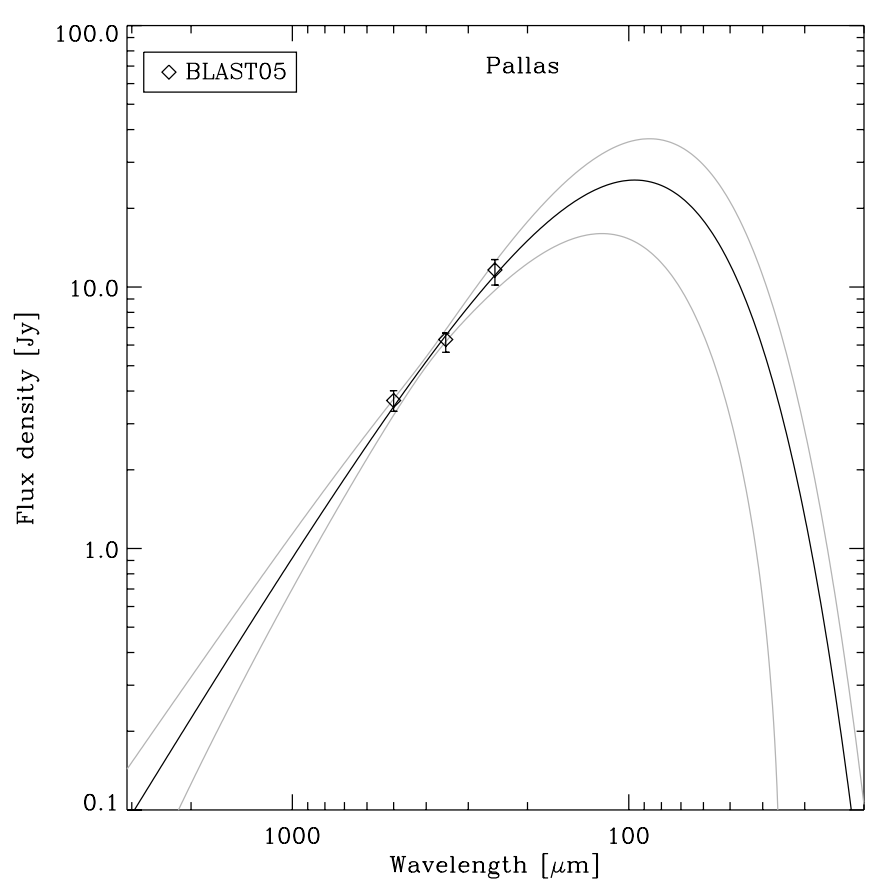

FIG. 4.-Pallas SED. Symbols and lines are as those described in Fig. 2.

BLAST, is $0.2 \pm 0.3$, which is the shallowest of all the sources described in this paper.

\section{4. $L 1014-I R S$}

LDN 1014 (Lynds 1962), which was classified as a "starless core" due to the lack of an FIR IRAS detection and no signature of a continuum outflow (Young et al. 2004), is a nearby dark cloud that contains one of the lowest luminosity protostellar systems, L1014-IRS. More recently, a bipolar molecular outflow has been discovered (Bourke et al. 2005). The distance to LDN 1014 is not firmly determined, with estimates of $\sim 200 \mathrm{pc}$ (Huard et al. 2006), 400-900 pc (Morita et al. 2006), and $<500$ pc (Shirley et al. 2007). The FIR-millimeter SED of L1014-IRS, derived from a physical model of a circumstellar disk heated by the central protostellar object, and constrained by recent Spitzer observations from 3 to $70 \mu \mathrm{m}$, predicts a strong spectral peak at $\sim 350 \mu \mathrm{m}$ (Young et al. 2004). Since L1014-IRS was unresolved by BLAST, we were unable to observe the predicted density profile. The BLAST fluxes at 350 and $500 \mu \mathrm{m}$, however, are in good agreement with the model but suggest that the SED (Fig. 5) peaks at shorter wavelengths, $<250 \mu \mathrm{m}$.

\subsection{K3-50}

K3-50 is a group of compact $\mathrm{H}$ II regions within the starforming complex W58, at a distance of about $8.5 \mathrm{kpc}$ (Peeters et al. 2002). Despite the nonoptimal PSF, BLAST clearly resolves at least two sources separated by $\simeq 2^{\prime}$, which can be identified as K3-50A and K3-50C (Howard et al. 1996). Figure 6 shows the $250 \mu \mathrm{m}$ image. Fluxes for K3-50A and K3-50C are obtained by simultaneously fitting to the BLAST data a model of two twodimensional Gaussian sources convolved with the BLAST PSFs, whose sizes, positions, and amplitudes are parameters in the fit. An alternative model of two point sources, fixed at the positions of K3-50A and K3-50C (from Howard et al. 1996), produces an indistinguishable solution for the inferred fluxes.

Thompson et al. (2006) report an integrated $450 \mu \mathrm{m}$ SCUBA flux of $256 \pm 79$ Jy for K3-50A, which is consistent with our 


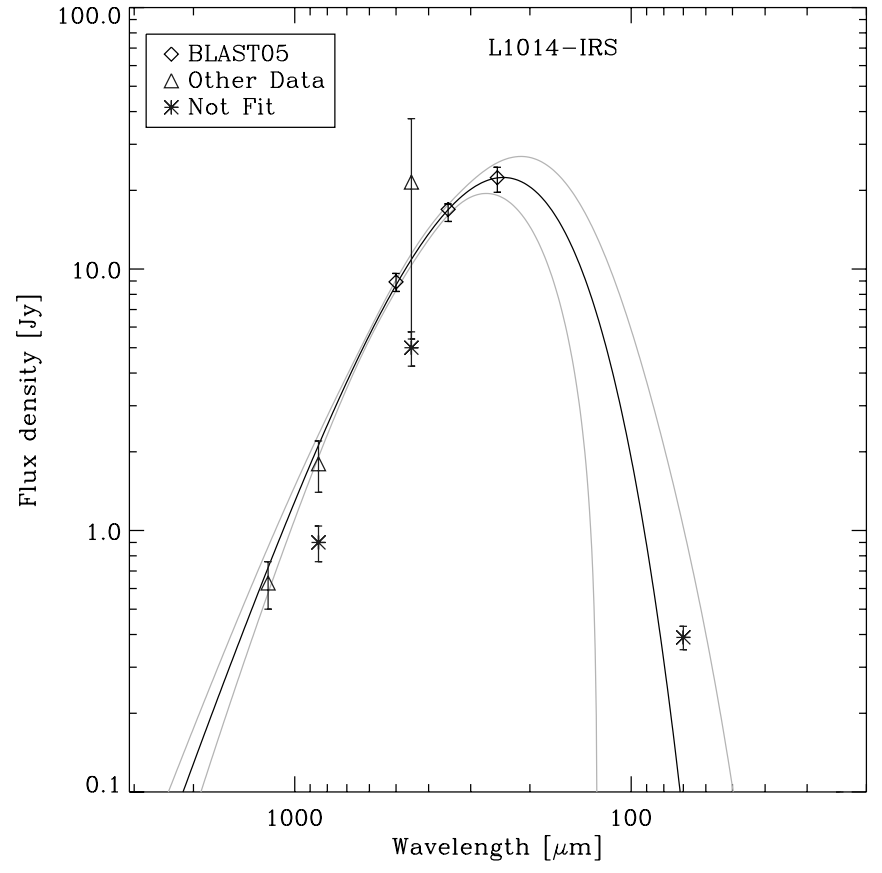

FIG. 5.-L1014-IRS SED. Symbols and lines are as those described in Fig. 2. The fit is poor, partly because a single-temperature modified blackbody may not be the best model, but also because of resolution issues with some of the photometry. We have not included the lower 450 and $850 \mu \mathrm{m}$ data points, which clearly disagree with the remaining photometry, probably because of resolution effects. Non-BLAST data are from Visser et al. (2002) and Young et al. (2004). [See the electronic edition of the Journal for a color version of this figure.]

$500 \mu \mathrm{m}$ photometry, despite issues with chopping and the restricted map size for SCUBA making the comparison potentially complicated. Although there is some suggestion in the literature that these sources may be variable, ${ }^{16}$ we fit their individual FIR-millimeter SEDs (Fig. 6) with single-temperature modified blackbody emission. K3-50C appears to have a dust temperature of only $9 \mathrm{~K}$, substantially colder than that in K3-50A, which has dust radiating at temperatures of $\sim 40 \mathrm{~K}$ (Table 3 ), in good agreement with previous estimates based on FIR Kuiper Airborne

\footnotetext{
16 See http://www.jach.hawaii.edu/JCMT/continuum/calibration/sens/ potentialcalibrators.html.
}

Observatory data (Thronson \& Harper 1979). This difference in dust temperature may be due to the fact that $\mathrm{K} 3-50 \mathrm{C}$ is embedded more deeply in the molecular cloud than K3-50A, or it may reflect the different evolutionary stages of the objects, with K3-50A generally agreed to be younger (Howard et al. 1996).

$$
\text { 5.6. } W 75 N
$$

The W75N (Westerhout 1958) and DR 21 field contains a set of young protostar/compact $\mathrm{H}$ II regions located in the Cygnus $\mathrm{X}$ molecular cloud complex. These sources are at a distance of about 3 kpc (Campbell et al. 1982; Pipenbrink \& Wendker 1988), although other estimates suggest a closer distance of around $1.7 \mathrm{kpc}$ (Jakob et al. 2007). The region has been extensively studied over a wide wavelength range, with many detailed spectroscopic (including $\mathrm{OH}$ and other maser lines) and continuum observations (see, e.g., Davis et al. 2007). Despite its extended emission, $\mathrm{W} 75 \mathrm{~N}$ has been suggested as a potential calibrator for submillimeter observations (Sandell 2003). The W75N region contains at least three pointlike objects that are resolved by BLAST: W75N, DR 21 (Downes \& Rinehart 1966), and DR 21 $(\mathrm{OH})$. Flux densities for $\mathrm{W} 75 \mathrm{~N}$ are obtained by the matched filter technique, as described in $\S 2$. Since DR $21(\mathrm{OH})$ and DR 21 overlap, their flux densities are derived from a model of point sources convolved with the BLAST PSF (as described in $\S 5.1$ ), where the positions and fluxes are the free parameters. These BLAST data suggest that DR 21 has a higher value of $\beta$ and a lower dust temperature than DR $21(\mathrm{OH})$, in agreement with what has been found in other studies (e.g., Jakob et al. 2007).

In addition to the detection of the protostellar cores within the $\mathrm{H}$ II regions, and as can be seen in Figure 7, the BLAST data also show significant extended surface brightness structure $\left(\sim 1000 \mathrm{MJy} \mathrm{sr}^{-1}\right.$ at $\left.250 \mu \mathrm{m}\right)$. This is highly correlated with the near-IR extinction maps toward the same region (Kumar et al. 2007), indicating that it is real, rather than being mapmaking artifacts.

\subsection{Mrk 231}

Mrk 231 (Markarian 1969), also known as IRAS 12540+5708, is the most luminous infrared galaxy within the local volume out to a distance of $175 \mathrm{Mpc}$. Mrk 231 hosts a Seyfert 1 nucleus, although the FIR emission is known to be dominated by star formation within a region that is of order $1^{\prime}$ (see Lípari et al. 2005 and references therein). It is therefore pointlike for BLAST,
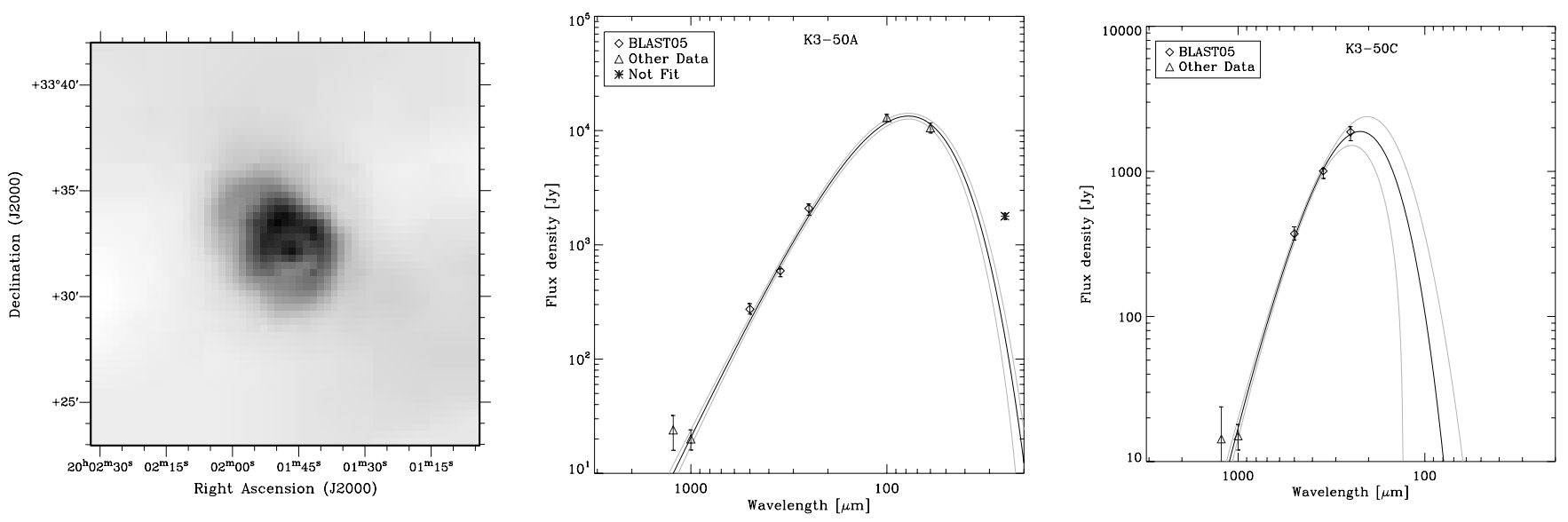

FIG. 6.-Left: $250 \mu \mathrm{m}$ image of the K3-50 region, with the brighter source K3-50A to the southwest and K3-50C to the northeast. SEDs for K3-50A (middle) and K3-50C

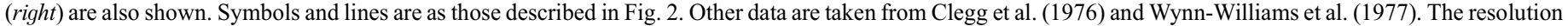

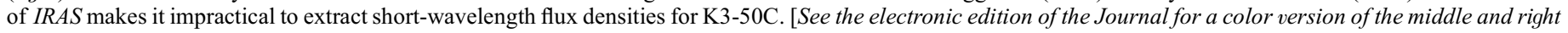
panels of this figure.] 

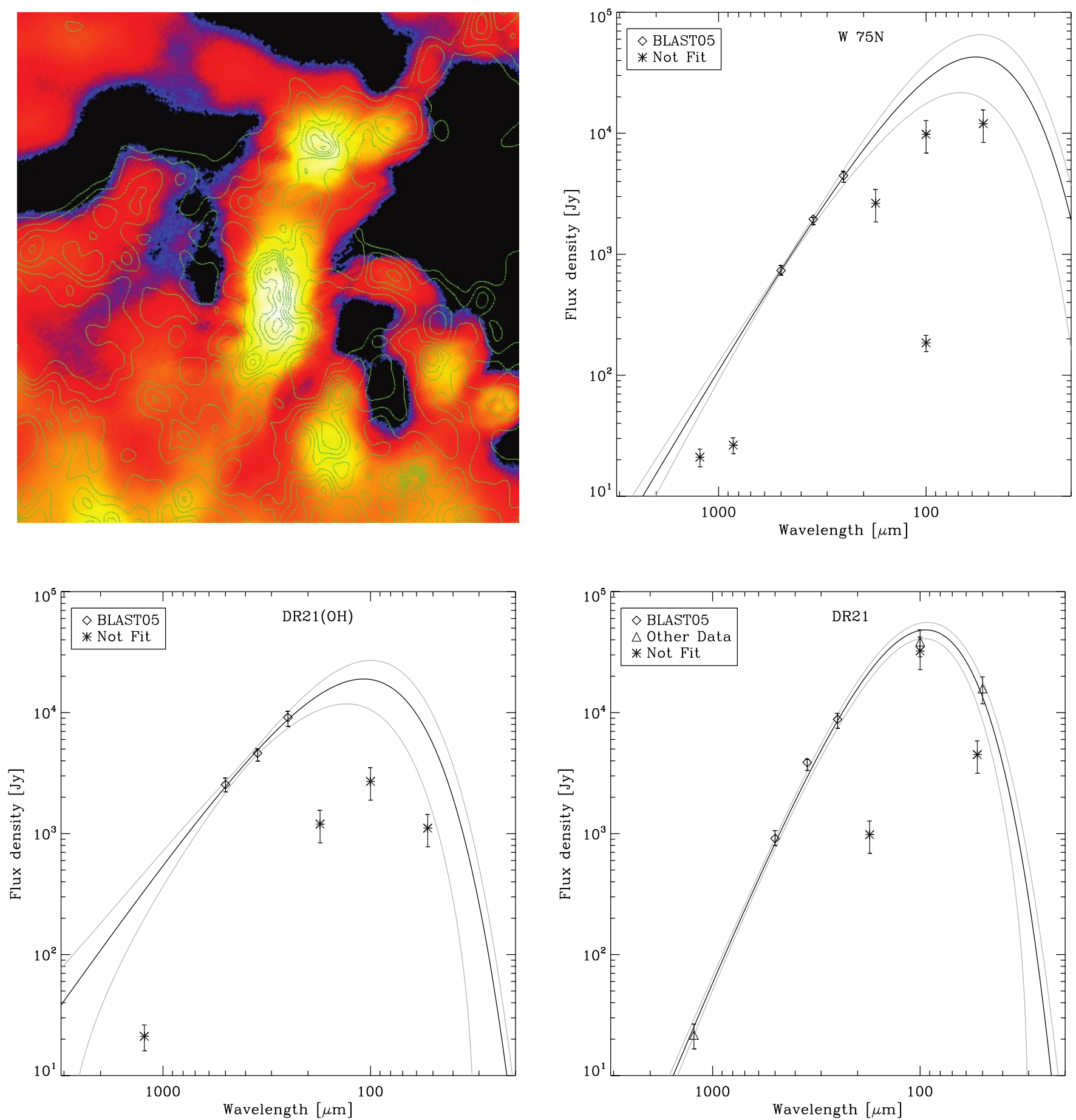

FIG. 7.-Top left: $250 \mu \mathrm{m}$ BLAST image of the W75N region, with green contours showing the distribution of IR extinction (Kumar et al. 2007). We divide the BLAST emission into three sources identified with W75N to the north; DR $21(\mathrm{OH})$, the northern part of the merged pair; and DR 21, the southernmost source. The $1^{\prime}$ pixel size in this map hides much of the structure in the BLAST PSF. SEDs show data and best-fitting models for W75N (top right), DR 21 (OH) (bottom left), and DR 21 (bottom right). Symbols and lines are as those described in Fig. 2. The data from Clegg et al. (1976), Davis et al. (2007), Helou \& Walker (1988), and Harvey et al. (1977) are not adopted in the fits because of the smaller aperture used to determine those flux density measurements. The data from Harvey et al. (1977) suggest a temperature of $65 \mathrm{~K}$ for W75N and $40 \mathrm{~K}$ for DR $21(\mathrm{OH})$, and these are used as constraints in our SED fits. Other data are from Clegg et al. (1976) and Colome et al. (1995). [See the electronic edition of the Journal for a color version of the top right, bottom left, and bottom right panels of this figure.]

given the measured PSF (Fig. 1). Mrk 231 has a well-measured SED and has been used as a template for photometric redshift estimates of extragalactic sources (e.g., Klaas et al. 2001; Aretxaga et al. 2005). There are useful FIR data from IRAS and ISO, although Klaas et al. (2001) describe the existing ISO photometry as "partly distorted and uncertain." Benford (1999) reports a measurement at $350 \mu \mathrm{m}$ of $1.05 \pm 0.25 \mathrm{Jy}$, which is consistent with the BLAST $350 \mu \mathrm{m}$ photometry. There is also $450 \mu \mathrm{m}$ photometry published, although using a much smaller aperture (Rigopoulou et al. 1996). BLAST flux densities thus help constrain the SED in 


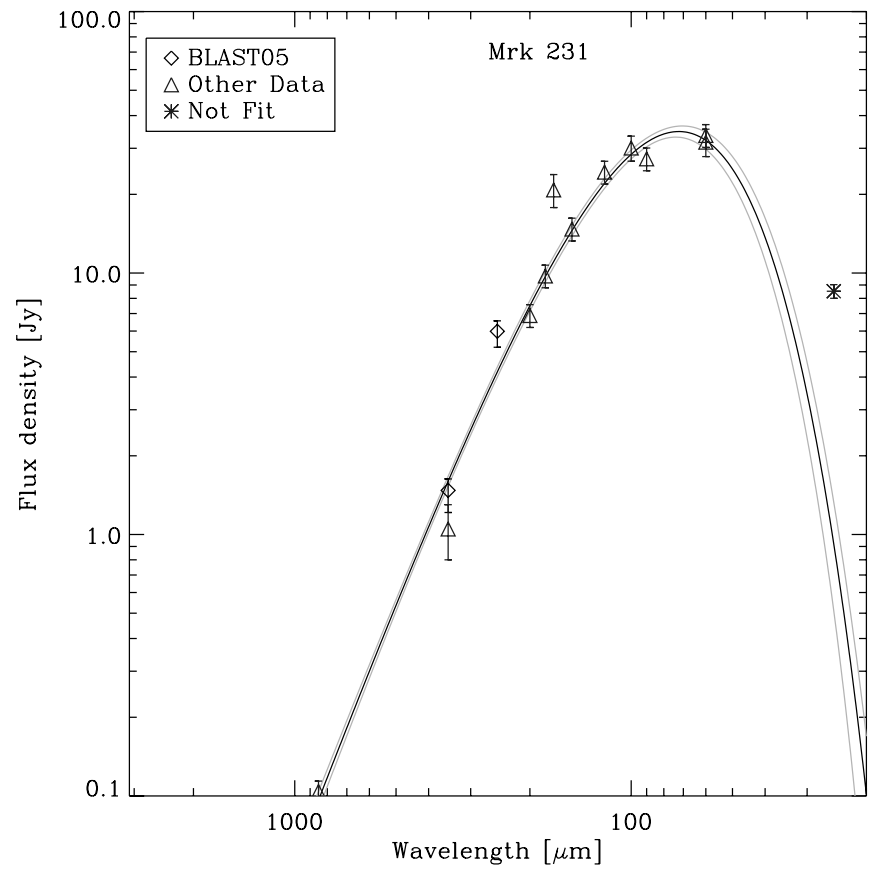

FIG. 8.-Mrk 231 SED showing data and best-fitting model. Symbols and lines are as those described in Fig. 2. Mrk 231 was not detected by BLAST at $500 \mu \mathrm{m}$. Other data are taken from Benford (1999), Klaas et al. (2001), Lisenfeld et al. (2000), Stickel et al. (2004), and Helou \& Walker (1988). [See the electronic edition of the Journal for a color version of this figure.] the poorly sampled submillimeter regime (see Fig. 8). However, given the relative faintness of this source, the improvement is modest compared with other sources reported here. We find slightly lower values for temperature and $\beta$ compared with Klaas et al. (2001), but they also claim that the data are better fitted with multiple temperature components.

\subsection{Compact Protostellar Sources}

The remaining Galactic targets, described below, are all compact protostellar sources, identified via color criteria in the FIR, that satisfy the BLAST selection requirements. We present here only their fluxes, dust masses, and luminosities (Tables 2 and 3) derived from the best-fit models describing their SEDs (Fig. 9).

\subsubsection{IRAS $20126+4104$}

IRAS $20126+4104$ is a high-mass compact protostar, located toward the Cygnus X molecular cloud complex, although its exact distance is uncertain. We adopt a distance of $1.7 \mathrm{kpc}$ (Shepherd et al. 2000). It is probably the best-studied example of a massive protostellar system associated with a disk and outflow (Edris et al. 2005). BLAST provides better constraints on the SED (Wilking et al. 1989; Hunter et al. 2000), particularly the submillimeter slope, as shown in Figure 9.

\subsubsection{IRAS $21078+5211$}

IRAS $21078+5211$ is a compact $\mathrm{H}$ II region with a water maser, located at a distance of approximately $1.65 \mathrm{kpc}$ (Molinari et al.
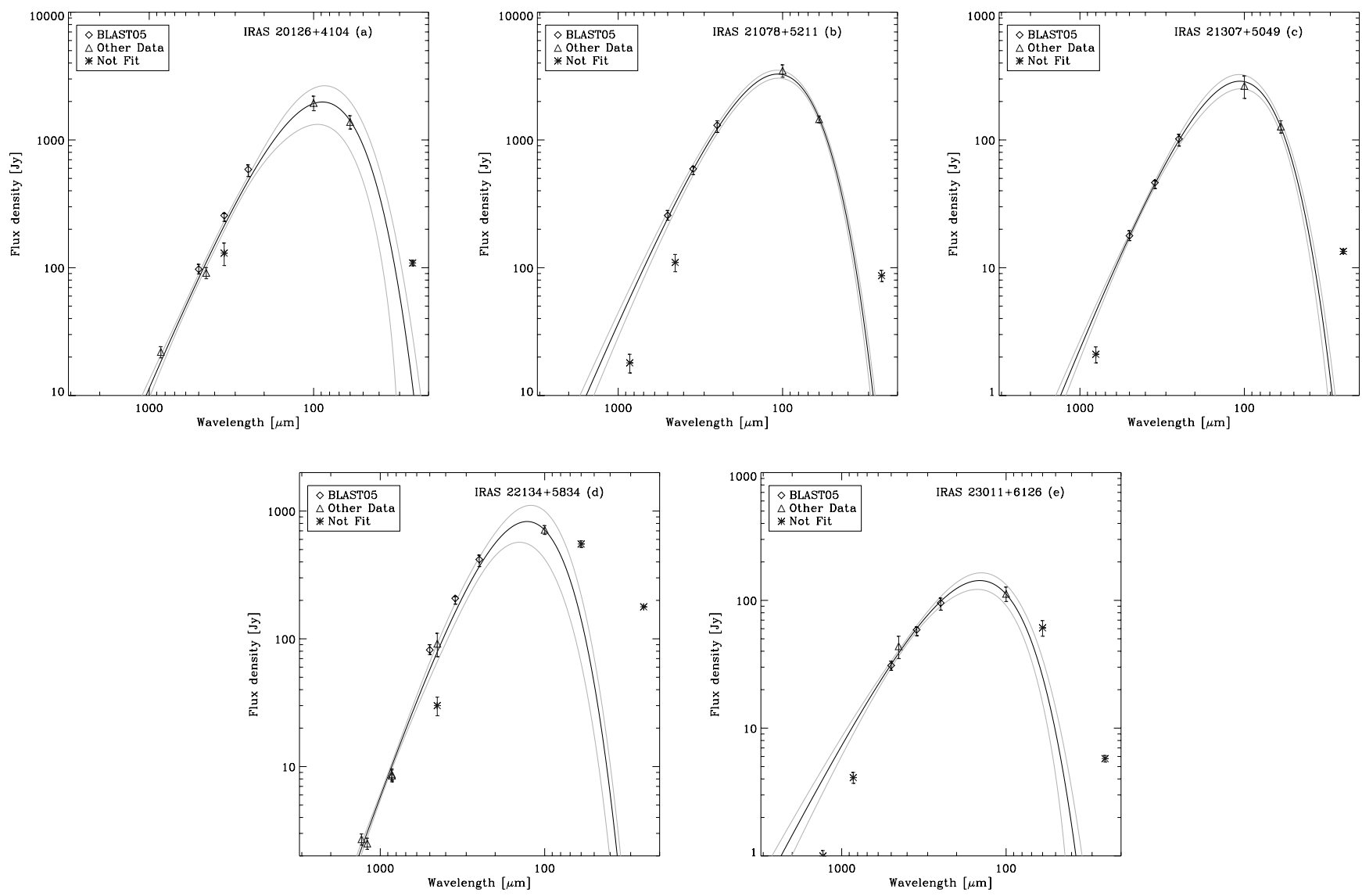

FIG. 9.- SEDs of compact protostellar sources, including the BLAST fluxes (see Table 1). The data symbols and fitted curves have the same meaning as those described in Fig. 5. (a) IRAS 20126+4104; additional data are taken from Helou \& Walker (1988), Hunter et al. (2000), Beuther et al. (2002), and Williams et al. (2004). The $350 \mu \mathrm{m}$ data point from Hunter et al. (2000) is not used in the fit, due to the smaller aperture. (b) IRAS 21078 +5211 ; additional data are taken from Helou \& Walker (1988) and Jenness et al. (1995). Three data points are not used in the fit due to the small effective aperture sizes in those measurements. (c) IRAS 21307+5049; additional data are taken from Molinari et al. (2000) and Helou \& Walker (1988). The $850 \mu$ m data point from Molinari et al. (2000) is not used in the fit due to its smaller aperture compared to the BLAST PSF. (d) IRAS 22134+5834; additional data are taken from Helou \& Walker (1988), Chini et al. (2001), Beuther et al. (2002), and Williams et al. (2004). (e) IRAS 23011+6126; additional data are taken from Helou \& Walker (1988) and Chini et al. (2001). The $850 \mu$ m data point from Chini et al. (2001) is not used in the fit due to the smaller aperture. [See the electronic edition of the Journal for a color version of this figure.] 
1996; Wouterloot et al. 1993). The best-fit single-temperature model with $T \simeq 34 \mathrm{~K}$ and $\beta \simeq 1.1$ is consistent with a previous study by McCutcheon et al. (1991).

\subsubsection{IRAS $21307+5049$}

IRAS $21307+5049$ (also Mol 136; Molinari et al. 1996) is a high-mass protostellar candidate. At a distance of $3.6 \mathrm{kpc}$, it has an angular size of about $4^{\prime \prime}$ in the millimeter (Molinari et al. 2002) and is therefore pointlike to BLAST. Fontani et al. (2004) present $850 \mu \mathrm{m}$ SCUBA continuum observations, detecting an extended halo of $40^{\prime \prime}$ diameter and a compact unresolved core. The extended halo is not detected by BLAST. We only fit a singletemperature modified blackbody curve to the cold core component. We find a temperature of $32 \mathrm{~K}$ and luminosity of $L_{\mathrm{FIR}} \simeq$ $4000 L_{\odot}$, in very good agreement with Molinari et al. (2000).

$$
\text { 5.8.4. IRAS } 22134+5834
$$

IRAS $22134+5834$ is an intermediate- to high-mass protostar located within the extensive $\mathrm{H}$ II region S134, in the constellation Cepheus. It is at a distance of approximately $900 \mathrm{pc}$ (Dobashi et al. 1994) and has a total luminosity of $\sim 1600 L_{\odot}$.

\subsubsection{IRAS $23011+6126$}

IRAS $23011+6126$ is a young protostar located at a distance of $\sim 730 \mathrm{pc}$ (Crawford \& Barnes 1970). IRAS 23011+6126 is pointlike to BLAST. Chini et al. (2001) discuss the current best SED (similar to IRAS $22134+5834$ above). The peak of the SED is at about $150 \mu \mathrm{m}$, as shown in Figure 9. It appears to have $T \simeq 28 \mathrm{~K}$, with a shallower slope $(\beta=0.6 \pm 0.2)$ than most of the other protostellar sources that BLAST observed.

\section{CONCLUSIONS}

For the BLAST 2005 flight, the out-of-focus PSFs significantly reduced the point-source sensitivity, and yet because this is such a difficult wave band to observe from the ground, the BLAST05 data still produced useful results. The submillimeter flux densities reported here agree well with other measurements, at least when the aperture sizes are consistent. However, BLAST05 uncer- tainties are typically much smaller than those of other measurements in the 250-500 $\mu \mathrm{m}$ regime.

A particular advantage of the BLAST data is the ability to constrain the emissivity index $\beta$, due primarily to the three separate submillimeter channels, observed simultaneously with the same telescope, and having a consistent calibration procedure performed across all three bands.

Arp 220 was adopted as the primary calibrator for the BLAST05 data. A useful cross-check is to note that the derived flux densities for Pallas were all within $1 \sigma$ of the values derived from the TPM (Müller \& Lagerros 2002).

By applying this careful calibration procedure, BLAST has been able to improve estimates of dust temperatures and masses for several relatively bright sources and also to provide a database of submillimeter measurements that may be useful for calibrating future instruments.

The BLAST collaboration acknowledges the support of NASA through grants NAG5-12785, NAG5-13301, and NNGO6GI11G, the Canadian Space Agency (CSA), Canada's Natural Sciences and Engineering Research Council (NSERC), and the UK Particle Physics and Astronomy Research Council (PPARC). We would also like to thank the Columbia Scientific Balloon Facility (CSBF) staff for their outstanding work, as well as T. G. Müller for valuable discussions on Pallas. L. O. acknowledges partial support by the Puerto Rico Space Grant Consortium and by the Fondo Istitucional para la Investigacion of the University of Puerto Rico. C. B. N. acknowledges support from the Canadian Institute for Advanced Research. This research has been enabled by the use of WestGrid computing resources. This research also made use of the SIMBAD database, operated at the Centre de Donées astronomiques de Strasbourg (CDS), Strasbourg, France, and the NASA/IPAC Extragalactic Database (NED), which is operated by the Jet Propulsion Laboratory, California Institute of Technology, under contract with the National Aeronautics and Space Administration.
Aretxaga, I., Hughes, D. H., \& Dunlop, J. S. 2005, MNRAS, 358, 1240

Arp, H. 1966, ApJS, 14, 1

Beelen, A., Cox, P., Benford, D. J., Dowell, C. D., Kovács, A., Bertoldi, F., Omont, A., \& Carilli, C. L. 2006, ApJ, 642, 694

Benford, D. J. 1999, Ph.D. thesis, Caltech

Beuther, H., Schilke, P., Menten, K. M., Motte, F., Sridharan, T. K., \& Wyrowski, F. 2002, ApJ, 566, 945

Bourke, T. L., Crapsi, A., Myers, P. C., Evans, N. J., II, Wilner, D. J., Huard, T. L., Jørgensen, J. K., \& Young, C. H. 2005, ApJ, 633, L129

Campbell, M. F., Niles, D., Nawfel, R., Hawrylycz, M., Hoffmann, W. F., \& Thronson, H. A., Jr. 1982, ApJ, 261, 550

Chapin, E. L., et al. 2008, ApJ, 681, 428

Chini, R., Ward-Thompson, D., Kirk, J. M., Nielbock, M., Reipurth, B., \& Sievers, A. 2001, A\&A, 369, 155

Clegg, P. E., Rowan-Robinson, M., \& Ade, P. A. R. 1976, AJ, 81, 399

Cohen, M., \& Kuhi, L. V. 1977, ApJ, 213, 79

Colome, C., Harvey, P. M., Lester, D. F., Campbell, M. F., \& Butner, H. M. 1995, ApJ, 447, 236

Coppin, K., et al. 2006, MNRAS, 372, 1621

2008, MNRAS, 384, 1597

Cox, P., et al. 1996, A\&A, 315, L265

Crampton, D., Cowley, A. P., \& Humphreys, R. M. 1975, ApJ, 198, L135

Crawford, D. L., \& Barnes, J. V. 1970, AJ, 75, 952

Davis, C. J., Kumar, M. S. N., Sandell, G., Froebrich, D., Smith, M. D., \& Currie, M. J. 2007, MNRAS, 374, 29

Do, T., Morris, M., Sahai, R., \& Stapelfeldt, K. R. 2005, BAAS, 37, 1161

Dobashi, K., Bernard, J.-P., Yonekura, Y., \& Fukui, Y. 1994, ApJS, 95, 419

\section{EFERENCES}

Downes, D., \& Rinehart, R. 1966, ApJ, 144, 937

Dunne, L., \& Eales, S. A. 2001, MNRAS, 327, 697

Eales, S., Lilly, S., Webb, T., Dunne, L., Gear, W., Clements, D., \& Yun, M. 2000, AJ, 120, 2244

Edris, K. A., Fuller, G. A., Cohen, R. J., \& Etoka, S. 2005, A\&A, 434, 213

Fontani, F., Cesaroni, R., Testi, L., Molinari, S., Zhang, Q., Brand, J., \& Walmsley, C. M. 2004, A\&A, 424, 179

Friesen, R. K., Johnstone, D., Naylor, D. A., \& Davis, G. R. 2005, MNRAS, 361, 460

Griffin, M. J., Bock, J. J., \& Gear, W. K. 2002, Appl. Optics, 41, 6543

Griffin, M. J., \& Orton, G. S. 1993, Icarus, 105, 537

Griffin, M. J., Swinyard, B. M., \& Vigroux, L. 2004, Proc. SPIE, 5487, 413

Hargrave, P., Waskett, T., Lim, T., \& Swinyard, B. 2006, Proc. SPIE, 6275, 36 Hargrave, P., et al. 2008, ApJ, submitted

Harvey, P. M., Campbell, M. F., \& Hoffmann, W. F. 1977, ApJ, 211, 786

Helou, G., \& Walker, D. W., eds. 1988, IRAS Catalogs: The Small Scale Structure Catalog (Washington, DC: GPO)

Howard, E. M., Pipher, J. L., Forrest, W. J., \& de Pree, C. G. 1996, ApJ, 460, 744 Huard, T. L., et al. 2006, ApJ, 640, 391

Hunter, T. R., Churchwell, E., Watson, C., Cox, P., Benford, D. J., \& Roelfsema, P. R. 2000, AJ, 119, 2711

Jakob, H., Kramer, C., Simon, R., Schneider, N., Ossenkopf, V., Bontemps, S., Graf, U. U., \& Stutzki, J. 2007, A\&A, 461, 999

Jenness, T., Scott, P. F., \& Padman, R. 1995, MNRAS, 276, 1024

Jenness, T., Stevens, J. A., Archibald, E. N., Economou, F., Jessop, N. E., \&

Robson, E. I. 2002, MNRAS, 336, 14

Johnstone, D., Boonman, A. M. S., \& van Dishoeck, E. F. 2003, A\&A, 412, 157 
Klaas, U., et al. 2001, A\&A, 379, 823

Kumar, M. S. N., Davis, C. J., Grave, J. M. C., Ferreira, B., \& Froebrich, D. 2007, MNRAS, 374, 54

Lípari, S., Terlevich, R., Zheng, W., Garcia-Lorenzo, B., Sanchez, S. F., \& Bergmann, M. 2005, MNRAS, 360, 416

Lisenfeld, U., Isaak, K. G., \& Hills, R. 2000, MNRAS, 312, 433

Lynds, B. T. 1962, ApJS, 7, 1

Markarian, B. E. 1969, Astrofizika, 5, 286

McCutcheon, W. H., Sato, T., Dewdney, P. E., \& Purton, C. R. 1991, AJ, 101, 1435

Mitchell, D. L., et al. 1996, Icarus, 124, 113

Molinari, S., Brand, J., Cesaroni, R., \& Palla, F. 1996, A\&A, 308, 573 . 2000, A\&A, 355, 617

Molinari, S., Testi, L., Rodríguez, L. F., \& Zhang, Q. 2002, ApJ, 570, 758

Morita, A., et al. 2006, PASJ, 58, L41

Müller, T. G., \& Lagerros, J. S. V. 2002, A\&A, 381, 324

Omont, A., et al. 1995, ApJ, 454, 819

Patanchon, G., et al. 2008, ApJ, 681, 708

Pascale, E., et al. 2008, ApJ, 681, 400

Peeters, E., et al. 2002, A\&A, 381, 571

Pipenbrink, A., \& Wendker, H. J. 1988, A\&A, 191, 313

Price, S. D., \& Murdock, T. L. 1983, AFGL-TR-0208 Environmental Research Papers, 161, 1

Rigopoulou, D., Lawrence, A., \& Rowan-Robinson, M. 1996, MNRAS, 278, 1049 Sandell, G. 1994, MNRAS, 271, 75

2003, in The Calibration Legacy of the ISO Mission, ed. L. Metcalfe et al. (ESA SP-481; Noordwijk: ESA), 439
Sanders, D. B., Mazzarella, J. M., Kim, D.-C., Surace, J. A., \& Soifer, B. T. 2003, AJ, 126, 1607

Schlegel, D. J., Finkbeiner, D. P., \& Davis, M. 1998, ApJ, 500, 525

Scott, D., et al. 2006, BAAS, 38, 1072

Scoville, N. Z., Yun, M. S., \& Bryant, P. M. 1997, ApJ, 484, 702

Shepherd, D. S., Yu, K. C., Bally, J., \& Testi, L. 2000, ApJ, 535, 833

Shirley, Y. L., Claussen, M. J., Bourke, T. L., Young, C. H., \& Blake, G. A. 2007, ApJ, 667, 329

Speck, A. K., Meixner, M., \& Knapp, G. R. 2000, ApJ, 545, L145

Spinoglio, L., Andreani, P., \& Malkan, M. A. 2002, ApJ, 572, 105

Stickel, M., Lemke, D., Klaas, U., Krause, O., \& Egner, S. 2004, A\&A, 422, 39

Thompson, M. A., Hatchell, J., Walsh, A. J., MacDonald, G. H., \& Millar, T. J. 2006, A\&A, 453, 1003

Thronson, H. A., Jr., \& Harper, D. A. 1979, ApJ, 230, 133

Turner, A. D., et al. 2001, Appl. Opt., 40, 4921

Visser, A. E., Richer, J. S., \& Chandler, C. J. 2002, AJ, 124, 2756

Westerhout, G. 1958, Bull. Astron. Inst. Netherlands, 14, 215

Wilking, B. A., Blackwell, J. H., Mundy, L. G., \& Howe, J. E. 1989, ApJ, 345, 257

Williams, S. J., Fuller, G. A., \& Sridharan, T. K. 2004, A\&A, 417, 115

Wouterloot, J. G. A., Brand, J., \& Fiegle, K. 1993, A\&AS, 98, 589

Wright, E. L. 2007, preprint (astro-ph/0703640)

Wynn-Williams, C. G., Matthews, K., Werner, M. W., Becklin, E. E., \& Neugebauer, G. 1977, MNRAS, 179, 255

Yao, L., Seaquist, E. R., Kuno, N., \& Dunne, L. 2003, ApJ, 588, 771

Young, C. H., et al. 2004, ApJS, 154, 396 